\title{
Evaluating Brightness and Spectral Properties of Click Beetle and Firefly Luciferases Using Luciferin Analogues: Identification of Preferred Pairings of Luciferase and Substrate for In Vivo Bioluminescence Imaging
}

Giorgia Zambito, ${ }^{1,2,3}$ Natasa Gaspar, ${ }^{1,2,4}$ Yanto Ridwan, ${ }^{1,2}$ Mary P. Hall, ${ }^{5}$ Ce Shi, ${ }^{6}$ Thomas A. Kirkland, ${ }^{6}$ Lance P. Encell, ${ }^{5}$ Clemens Löwik, ${ }^{1,2,7}$ Laura Mezzanotte ${ }^{1,2}$

${ }^{1}$ Erasmus Medical Center, Radiology and Nuclear Medicine, Rotterdam, The Netherlands

${ }^{2}$ Erasmus Medical Center, Molecular Genetics, Rotterdam, The Netherlands

${ }^{3}$ Medres medical research $\mathrm{GmBH}$, Cologne, Germany

${ }^{4}$ Percuros B.V, Leiden, The Netherlands

${ }^{5}$ Promega Corporation, Madison, WI, USA

${ }^{6}$ Promega Biosciences Incorporated, San Luis Obispo, CA, USA

${ }^{7}$ CHUV Department of Oncology, University of Lausanne, Lausanne, Switzerland

\begin{abstract}
Purpose: Currently, a variety of red and green beetle luciferase variants are available for bioluminescence imaging (BLI). In addition, new luciferin analogues providing longer wavelength luminescence have been developed that show promise for improved deep tissue imaging. However, a detailed assessment of these analogues (e.g., Akalumine-HCl, CycLuc1, and amino naphthyl luciferin $\left(\mathrm{NH}_{2}-\mathrm{NpLH} 2\right)$ ) combined with state of the art luciferases has not been performed. The aim of this study was to evaluate for the first time the in vivo brightness and spectral characteristics of firefly (Luc2), click beetle green (CBG99), click beetle red 2 (CBR2), and Akaluc luciferases when paired with different D-luciferin (D-LH2) analogues in vivo.

Procedures: Transduced human embryonic kidney (HEK 293T) cells expressing individual luciferases were analyzed both in vitro and in mice (via subcutaneous injection). Following introduction of the luciferins to cells or animals, the resulting bioluminescence signal and photon emission spectrum were acquired using a sensitive charge-coupled device (CCD) camera equipped with a series of band pass filters and spectral unmixing software.

Results: Our in vivo analysis resulted in four primary findings: (1) the best substrate for Luc2, CBG99, and CBR2 in terms of signal strength was D-luciferin; (2) the spectra for Luc2 and CBR2 were shifted to a longer wavelength when Akalumine- $\mathrm{HCl}$ was the substrate; (3) CBR2 gave the brightest signal with the near-infrared substrate, $\mathrm{NH}_{2}-\mathrm{NpLH} 2$; and (4) Akaluc was brighter when paired with either CycLuc1 or Akalumine-HCl when paired with D-LH2.
\end{abstract}

Electronic supplementary material The online version of this article (https:// doi.org/10.1007/s11307-020-01523-7) contains supplementary material, which is available to authorized users. 
Conclusion: We believe that the experimental results described here should provide valuable guidance to end users for choosing the correct luciferin/luciferase pairs for a variety of $\mathrm{BLI}$ applications.

Key words: Bioluminescence, In vivo imaging, Luciferase, Emission spectrum, Luciferin

\section{Introduction}

Bioluminescence imaging (BLI) is a well-known, noninvasive technique employed during preclinical studies to track cells and monitor biological processes in living animals [1-3]. BLI is performed by capturing the light generated by a luciferase upon exogenous substrate (e.g., D-luciferin (DLH2)) addition to report real-time, cellular, and molecular events [4].

Over the last decade, the bioluminescence toolbox has greatly expanded $[1,5,6]$. Novel luciferin analogues have been introduced that enhance light emission in vivo and increase detection sensitivity in deeper tissues [7]. Cycluc1 has been shown to enhance emission of codon optimized firefly luciferase (Luc2), especially in the brain. Furthermore, this system provides slightly red-shifted emission resulting in deeper light penetration and less scattering of the bioluminescence signal $[8,9]$. Likewise, Akalumine- $\mathrm{HCl}$ has a spectral peak in the near infrared (NIR) $(677 \mathrm{~nm})$ and enhanced emission with Luc2 when administered at low concentration [10]. Akalumine-HCl paired with the recently engineered Akaluc luciferase is even brighter, although the spectral peak is blue-shifted to $650 \mathrm{~nm}$ [11]. Amino naphthyl luciferin $\left(\mathrm{NH}_{2}-\mathrm{NpLH} 2\right)$ represents another new substrate with potential for deeper tissue BLI [12]. This substrate was shown to emit in the NIR with a peak of $740 \mathrm{~nm}$ when reacting with an engineered version of click-beetle luciferase (CBR2). CBR2 can also utilize D-LH2 and this combination was shown to improve imaging in black fur mice compared with Luc2/ D-LH2.

Research into the development of improved BLI reagents has generally focused on bioluminescence systems comprised of compatible luciferase/luciferin pairings [13-18]. Most comparative studies have been performed using DLH2. For example, Miloud et al. compared firefly (Luc2) and click beetle luciferases in vivo with D-LH2 as substrate and concluded that click beetle green (CBG99) has sensitivity and total photon yield comparable with click beetle red [15]. In other studies, Luc2 was shown to have improved performance compared with a red-shifted firefly mutant (PpyRE9) and CBG99 for brain imaging [16, 17], but D-LH2 was the only substrate examined. A direct comparison (either in vitro or in vivo) of emission spectra and relative brightness of bioluminescence systems comprised of different luciferase enzymes in combination with novel luciferins has, to date, not been reported.

Here, we provide a detailed in vitro and in vivo analysis of brightness and emission spectra for four luciferases when combined with four different substrates using a CCD camera equipped with a series of band pass filters and spectral unmixing software. We anticipate that the results of this comparative analysis will help enable researchers to choose the best enzyme/substrate pairs for different BLI applications. In addition, our findings revealed that depending on the luciferase/luciferin pair, a wide range of spectral emission peaks (i.e., multicolored luciferases) is available that could broaden the BLI toolbox in the future for multiplex analysis both in vitro and in vivo.

\section{Materials and Methods}

\section{Animals}

Animal experiments were approved by the Bioethics Committee of Erasmus MC, Rotterdam, The Netherlands, and performed in accordance with national guidelines and regulations established by the Dutch Experiments on Animal Act $(\mathrm{WoD})$ and by the European Directive on the Protection of Animals used for scientific purpose (2010/63/EU). BALB/C nude (females) were obtained from Charles River Laboratory (The Netherlands). All mice aged 6-8 weeks were provided access to food and water ad libitum and were hosted in the animal facility at the Erasmus MC, Rotterdam, The Netherlands.

\section{Cell Line}

Human embryonic kidney cells (HEK 293T) were cultured in Dulbecco's Modified Eagle's Medium (DMEM) (Sigma, St. Louis, MO, USA) supplemented with $10 \%$ of FBS and $1 \%$ Penicillin-Streptomycin. The culture was incubated at $37{ }^{\circ} \mathrm{C}$ with $5 \% \mathrm{CO}_{2}$.

\section{Lentivirus Production}

Virus production and cell transduction were performed under appropriate biosafety level conditions (ML-II) in accordance with the National Biosafety Guidelines and Regulations for Research on Genetically Modified Organisms. Procedures and protocols were reviewed and approved by the EMC Biosafety Committee (GMO permit 99-163). The lentiviral plasmids pCDH-EF1-CBG99-T2A-copGFP, pCDH-EF1-Luc2-T2A-copGFP, and pCDH-EF1-CBR2T2A-copGFP were previously described $[12,15]$. The plasmid pCDH-EF1-Akaluc-T2A-copGFP was produced by 
inserting the sequence of Akaluc (amplified with specific primers from pcDNA3 Venus-Akaluc plasmid from RIKEN BRC repository) without stop codon using BamHI and NotI sites in pCDH-EF1-MCS-T2A-copGFP vector. Lentiviruses were produced by transfection of HEK 293T packaging cells with three packaging plasmids (pCMV-VSVG, pMDLgRRE, pRSV-REV; Addgene, Cambridge, MA, USA) and the lentiviral vector plasmids as previously described in details [16].The supernatant containing lentiviral particles were collected after 48 and $72 \mathrm{~h}$. Subsequent quantification of the virus was performed using a standard antigen-capture HIV p24 ELISA (ZeptoMetrix Corporation, NY, USA).

\section{Cell Transduction and Transfection}

Cell transduction was performed by culturing HEK 293T cells in DMEM supplemented with $10 \%$ of FBS and $1 \%$ of Penicillin-Streptomycin at the density of 200,000 cells in a T25-flask with $5 \mathrm{ml}$ of medium. Expression in the lentiviral plasmid is driven by housekeeping elongation factor $1 \alpha$ (EF1) promoter. Cells were transduced with MOI 1 of either pCDH-EF1-Luc2-T2A-copGFP, pCDH-EF1-CBG99-T2AcopGFP, pCDH-EF1-CBR2-T2A-copGFP, or pCDH-EF1Akaluc-T2A-copGFP lentivirus plus with polybrine (hexametride bromide, Sigma-Aldrich) at the final concentration of $8 \mu \mathrm{g} / \mathrm{ml}$. Transgene expression was confirmed by the presence of the super bright green fluorescent protein copGFP from the copepod Potentilla plumata (excitation/ emission maximum $=482 / 502 \mathrm{~nm}$ ).

\section{Flow Cytometry to Sort Stable Cell Lines}

Positive stable clones were sorted for comparable levels of copGFP expression by cell sorting (BD-FACS ARIA III, BD Biosciences). Forward and side scatters were also drawn to eliminate cellular debris from the analysis and to select highly positive cells for GFP.

\section{In Vitro BLI}

Transduced cells were plated at a density of $2 \times 10^{4}$ cells per well in a black 96-well plate (Greiner Cell Star ${ }^{\circledR}$ ) and imaged in $100 \mu \mathrm{l}$ of D-PBS. Bioluminescence signal from wells was measured with IVIS ${ }^{\circledR}$ spectrum system (PerkinElmer, Boston, MA, USA) every $5 \mathrm{~min}$ after substrate addition (final concentration of each substrate was $0.1 \mathrm{mM}$ ). All in vitro measurements were acquired after 1 min at $37^{\circ} \mathrm{C}$ using a 30 -s acquisition time with an open filter or using a series of band pass filters ranging from 520 to $800 \mathrm{~nm}$. Data were analyzed by the Living Image software version 4.3 (PerkinElmer). Data in every well were normalized for fluorescence emission detected using a GloMax ${ }^{\circledR}$-Multi plate reader.

\section{In Vivo BLI}

Each stable expressing cell line was injected subcutaneously $1 \times 10^{5}$ cells $/ 50 \mu \mathrm{l}$. The number of animals was chosen according to power analysis ( $p$ value at least $<0.05$ and power $95 \%$ ) considering that we expected from the data generated in vitro that the brightest BL system would differ by $1-2$ orders of magnitude in vivo. Mice ( $N=3$ per group) received two different cell lines, one in each flank. Animals were then imaged after intraperitoneal injection of D-LH2 substrate $(150 \mathrm{mg} / \mathrm{kg}), \mathrm{NH}_{2}-\mathrm{NpLH} 2$ substrate $(220 \mathrm{mg} / \mathrm{kg})$, CycLuc1 $(7.6 \mathrm{mg} / \mathrm{kg})$, and Akalumine- $\mathrm{HCl}$ substrate $(50 \mathrm{mg} / \mathrm{kg})$. These doses were chosen based on maximum solubility (for $\mathrm{CycLuc1}$ and Akalumine- $\mathrm{HCl}$ ), tolerability in mice, and maximum attainable signal based on previous findings. Mice were randomly assigned and anesthetized by isoflurane inhalation prior to performing BLI imaging. The person performing the subcutaneous injections was blind as to the cells being injected. Images were acquired with the IVIS $\AA$ spectrum small animal imager system (PerkinElmer). Light was measured using open filter and a series of $20 \mathrm{~nm}$ wavelength band filters from 520 to $800 \mathrm{~nm}$ with acquisition time of $30 \mathrm{~s}$ during a time of about $30 \mathrm{~min}$ after substrate injection (kinetic analysis). Emission signals were measured with the Living Image software ${ }^{\circledR}$ version 4.3 (Perkin Elmer).

\section{Statistical Analysis}

All statistical analyses were performed using the GraphPad Prism 6 software and one-way ANOVA followed by Tukey's post-test. $p$ values $<0.05$ were considered statistically significant.

\section{Results}

In Vitro Evaluation of Emission Properties for Different Combinations of Luciferase Variant and Luciferin Analogue

The aim of this study was to evaluate in vitro and in vivo light emission and spectral differences between four luciferases (Luc2, CBG99, CBR2, and Akaluc) when combined with D-LH2 or three luciferin analogues $\left(\mathrm{NH}_{2}-\mathrm{NpLH} 2\right.$, Akalumine- $\mathrm{HCl}$, or CycLuc1) for bioluminescence imaging (BLI). To compare the different emissions, HEK 293T cells stably expressing each of the four luciferases were treated with substrates $(0.1 \mathrm{mM})$ and imaged at $37{ }^{\circ} \mathrm{C}$. Equimolar expression of each luciferase was achieved by selecting cells for GFP emission.

We found that the luciferase/luciferin pairs yielding the highest photon emission $(p$ value $<0.001)$ were Luc2/D-LH2 and Akaluc/CycLucl when the substrate was added at a concentration of $0.1 \mathrm{mM}$. The combinations of Luc2/CycLuc1, Akaluc/Akalumine-HCl, CBG99/D-LH2, 
and CBR2/D-LH2 produced $\sim 2$-fold fewer photons (Fig. 1a), while cells expressing CBG99 were much less efficient ( 100-fold dimmer with $\mathrm{NH}_{2}-\mathrm{NpLH} 2 /$ Akalumine-HCl; 10 fold dimmer with CycLuc1) (Fig. 1a). CBR2-expressing cells were more promiscuous compared with CBG99 cells. However, they generated 10-fold less luminescence (compared with Luc2/D-LH2) with Akalumine- $\mathrm{HCl}$ and $\mathrm{NH}_{2}-$ NpLH2. The CBR2-expressing cells gave a signal

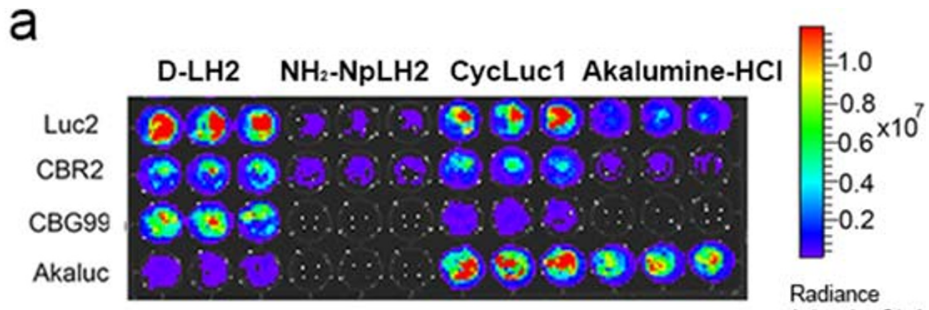

b
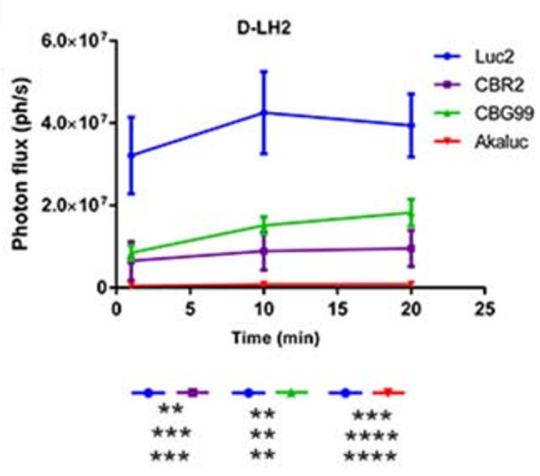

d
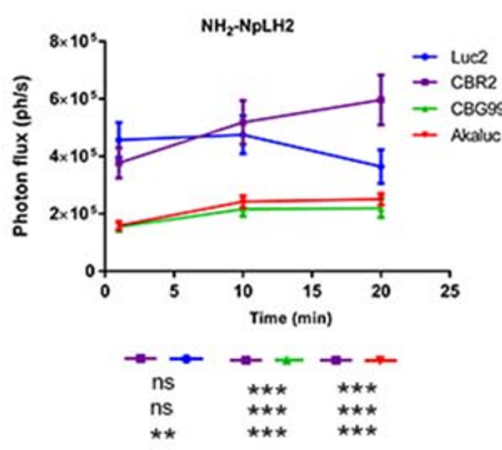

C
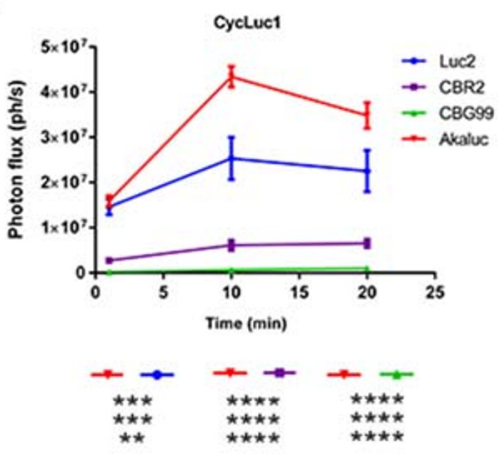

e

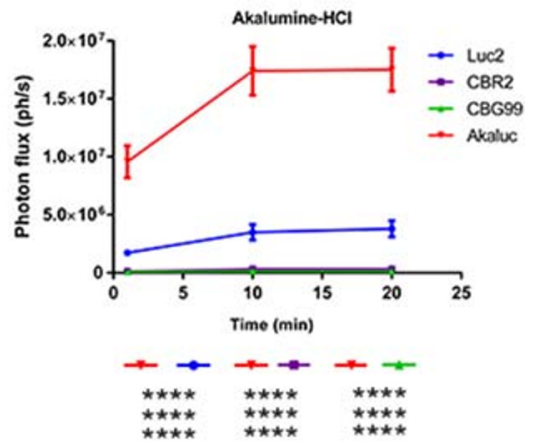

$f$

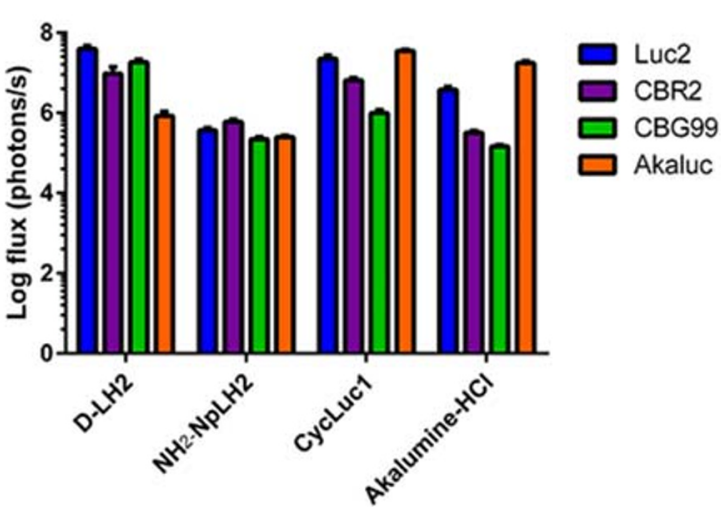

Fig. 1. (a) Bioluminescence profiles for Luc2, CBR2, CBG99, and Akaluc luciferases combined with four different luciferin analogues in live cells. (b-f) Photon flux (ph/s) in HEK 293T cells expressing individual luciferases upon addition of substrates $(0.1 \mathrm{mM})$ was quantified using an exposure time of $30 \mathrm{~s}$. Statistical analysis $(N=3)$ was performed using one-way ANOVA followed by Tukey's $T$ test ( ${ }^{*} p<0.01$ for Luc2/D-LH2 compared with all combinations with the exception of Akaluc/Cycluc1 which was not significantly different). 

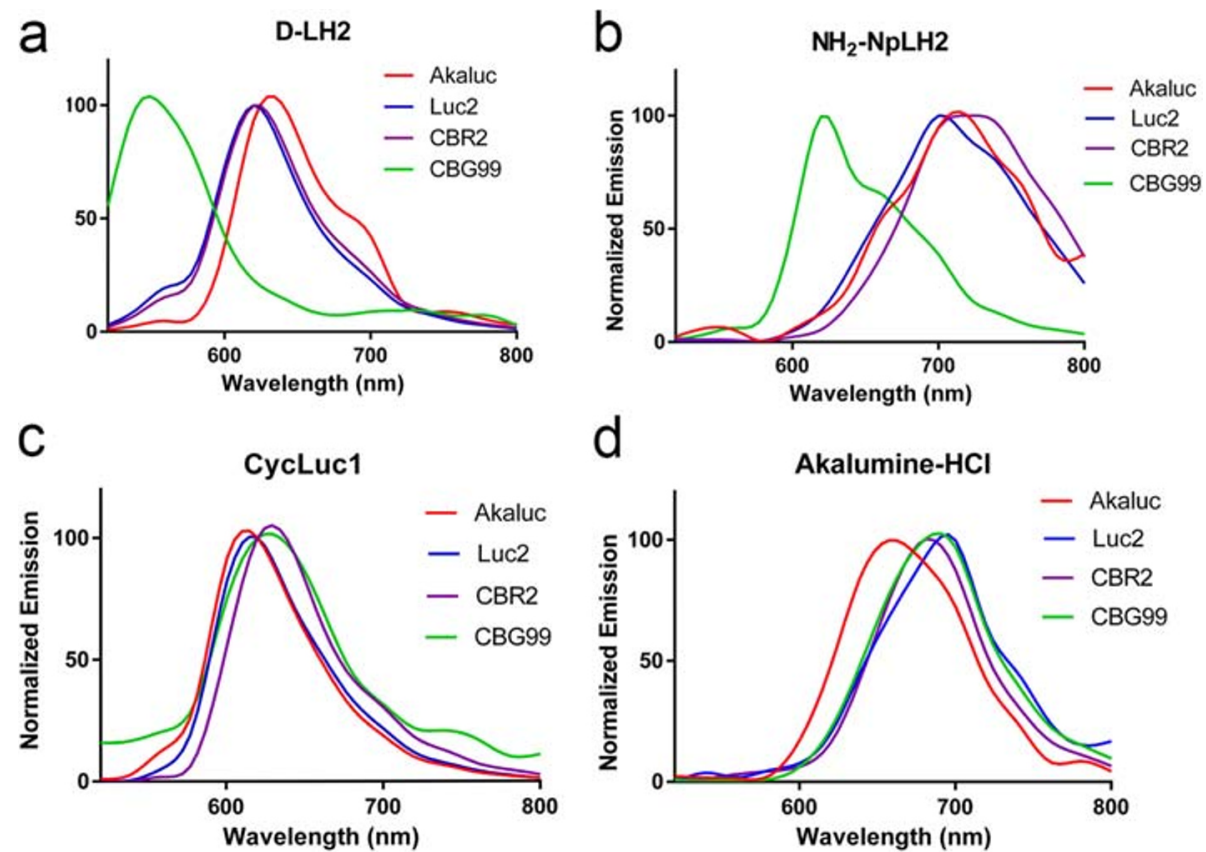

Fig. 2. In vivo (BALB/C) emission spectra for different combinations of luciferase (Luc2, CBG99, CBR2, or Akaluc; expressed in HEK393T cells implanted subcutaneously in the flanks) and luciferin or luciferin analogue. (a) D-LH2 (150 mg/kg), (b) $\mathrm{NH}_{2}{ }^{-}$ NpLH2 (220 mg/kg), (c) CycLuc1 $(7.6 \mathrm{mg} / \mathrm{kg})$, and (d) Akalumine-HCl (50 mg/kg); substrates were injected intraperitoneally). Spectral data was acquired 15-20 min after injection.

comparable with Luc2/D-LH2 with CycLuc1. Finally, Akaluc produced similar luminescence intensity when either Akalumine-HCl or CycLuc1 was used as substrate (Fig. 1a). Akaluc also showed nearly 100-fold lower signal with DLH2 or $\mathrm{NH}_{2}-\mathrm{NpLH} 2$ compared with Akalumine- $\mathrm{HCl}$ and CycLuc1.

\section{In Vivo Emission Spectrum of Luciferases Detected Using a Series of $20 \mathrm{~nm}$ Band Pass Filters}

The day of injection, HEK 293T cells (expressing the various luciferases) were prepared at a concentration of $2 \times 10^{6}$ cells $/ \mathrm{ml}$ in PBS and fluorescence emission measured at IVIS, confirming the comparable average expression of GFP (Supplementary Fig. 1). Following subcutaneous injection of $1 \times 10^{5}$ HEK 293T cells (expressing the various luciferases) into both flanks of mice, images were acquired after injection of D-LH2 $(150 \mathrm{mg} / \mathrm{kg}), \mathrm{NH}_{2^{-}}$ NpLH2 (220 mg/kg), Akalumine-HCl (50 mg/kg), or CycLuc1 $(7.6 \mathrm{mg} / \mathrm{kg})$. We used the optimal concentration for each given substrate based on previous literature $[8,10,12,19]$. For D-LH2, this was $150 \mathrm{mg} / \mathrm{kg}$ [19]. Because of poor aqueous solubility, CycLucl and Akalumine- $\mathrm{HCl}$ were injected at $7.6 \mathrm{mg} / \mathrm{kg}(5 \mathrm{mM}$ in saline) and $50 \mathrm{mg} / \mathrm{kg}$ (33 mM in saline), respectively [8, 10]. We previously demonstrated that the solubility of $\mathrm{NH}_{2}-\mathrm{NpLH} 2$ allows injection of a maximal dose of $220 \mathrm{mg} / \mathrm{kg}(60 \mathrm{mM}$ in saline) and that it produces significantly higher photon fluxes than a dose of $150 \mathrm{mg} / \mathrm{kg}$ [12]. Multiple acquisitions using a series of
$20 \mathrm{~nm}$ band pass filters were performed with an exposure time of $30 \mathrm{~s}$. The BLI measurements were performed at the time of peak of emission after injection of the luciferins into sedated animals.

In terms of emission spectra, Luc2/D-LH2, CBG99/DLH2, CBR2/D-LH2, and Akaluc/D-LH2 produced peaks at $610 \mathrm{~nm}, 540 \mathrm{~nm}, 620 \mathrm{~nm}$, and $640 \mathrm{~nm}$, respectively (Fig. 2a). $\mathrm{NH}_{2}-\mathrm{NpLH} 2$ caused a red shift of the peak of emission with all the luciferases (Luc2, $700 \mathrm{~nm}$; Akaluc, $720 \mathrm{~nm}$; CBR, $730 \mathrm{~nm}$; and CBG99, $620 \mathrm{~nm}$ ) (Fig. 2b). In contrast, when CycLucl was used as a substrate, the emission peak for each luciferase was in the range of $620 \mathrm{~nm}$ (Luc2 and Akaluc were green shifted towards $600 \mathrm{~nm}$ and CBG99 and CBR2 were red-shifted towards $640 \mathrm{~nm}$ ) (Fig. 2c). Akaluc/ Akalumine- $\mathrm{HCl}$, also referred to as the AkaBLI system [11], produced a peak of emission at $660 \mathrm{~nm}$ while the other luciferases peaked in the NIR $(\sim 680 \mathrm{~nm})$ when paired with Akalumine-HCl (Fig. 2d).

\section{In Vivo Comparison of Brightness of Luciferasel Luciferin Pairing}

Next, we compared the total emission of each luciferase in vivo with $\mathrm{D}-\mathrm{LH} 2$ or the luciferin analogues. Figure 3 shows the representative bioluminescent images of nude mice where CBG99, Luc2, CBR2, and Aka-Luc-expressing cells were implanted, and each of the different substrates was injected intraperitoneally. The data in Fig. 4 represents signals at peak of emission which differs slightly between 


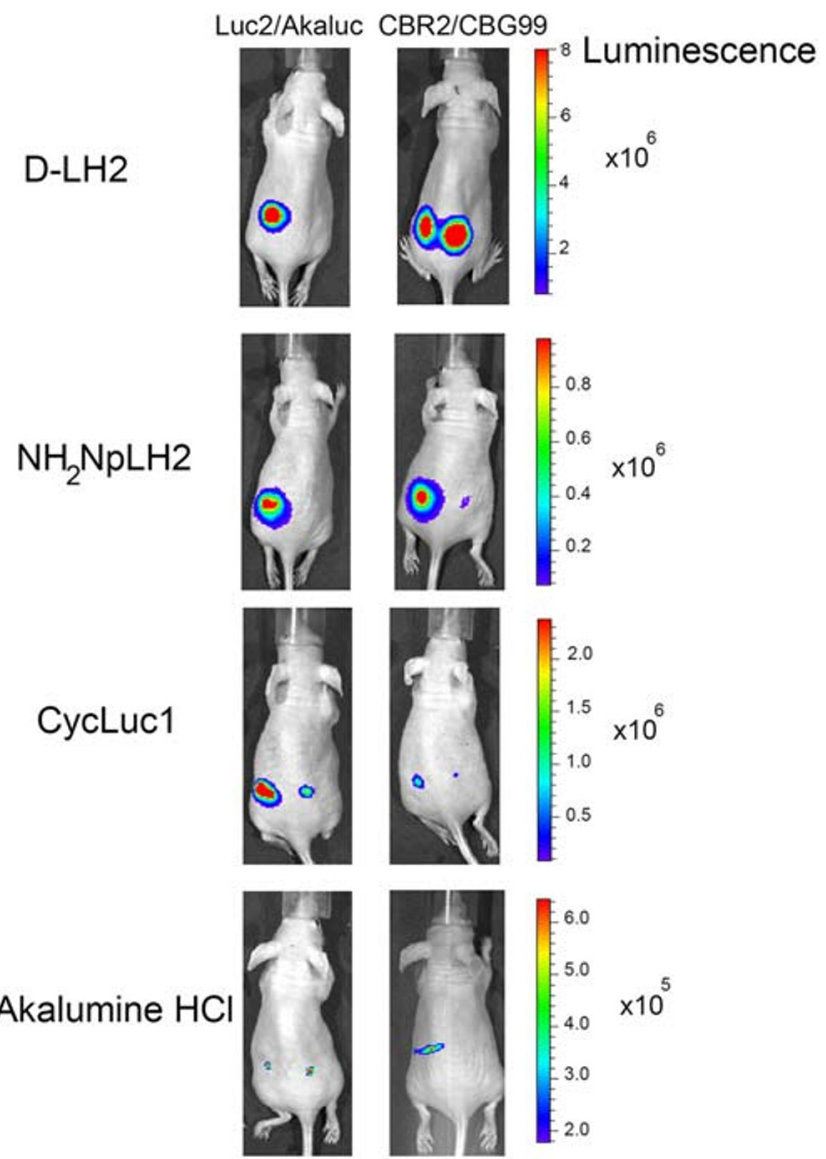

Radiance

$\left(\mathrm{p} / \mathrm{sec} / \mathrm{cm}^{2} / \mathrm{sr}\right)$

Fig. 3. Superficial bioluminescence imaging of BALB/C mice in which $1 \times 10^{5}$ HEK293T cells transduced with Luc2 and Akaluc or CBR2 and CBG99 were implanted subcutaneously into the left and right flanks of mice, respectively, and treated (intraperitoneally) with (a) D-LH2 $(150 \mathrm{mg} / \mathrm{kg})$, (b) $\mathrm{NH}_{2}-\mathrm{NpLH} 2(220 \mathrm{mg} / \mathrm{kg})$, (c) CycLuc1 $(7.6 \mathrm{mg} / \mathrm{Kg})$, and (d) Akalumine-HCl $(50 \mathrm{mg} / \mathrm{kg})$. Imaging data was collected using open filters and with an exposure time of $30 \mathrm{~s}$. Average luminescence is reported as photons $/ \mathrm{s} / \mathrm{cm}^{2} / \mathrm{sr}$.

BLI systems (Supplementary Fig. 2). Luc2, CBG99, and CBR2 paired with D-LH2 produced the highest signals which were 20-fold higher compared with Akaluc/D-LH2 ( $p$ value $<0.001$ ) (Fig. 4a). When $\mathrm{NH}_{2}-\mathrm{NpLH} 2$ was used as a substrate, Luc2 and CBR2 produced approximately 10-fold higher signal output ( $p$ value, 0.001 ) compared with both CBG99 and Akaluc (Fig. 4b). When CycLuc1 was used as a substrate, the strongest signal was detected for Luc2/Cycluc1. Akaluc, CBR2, and CBG99 paired with Cycluc1 produced $\sim 5,16$, and 70 -fold lower signal output, respectively (Fig. 4c). When Akalumine- $\mathrm{HCl}$ was used as a substrate, Luc2 and Akaluc produced $\sim 2$-fold higher signal compared with CBR2, and there was no detectable signal for CBG99 (Fig. 4d).
The luciferase/luciferin pairs that gave the highest photon yields in vivo were Luc2/D-LH2, Luc2/CycLuc1, CBG99/DLH2, and CBR2/D-LH2 $\left(1-2 \times 10^{7} \mathrm{ph} / \mathrm{s}\right)$. The following luciferin/luciferase pairs produced approximately 10-fold fewer photons: Akaluc/Cycluc1, Akaluc/Akalumine-HCl, Luc2/Akalumine-HCl, Luc2/ $\mathrm{NH}_{2}-\mathrm{NpLH} 2$, and CBR2/NH2NpLH2. Finally, the following pairs produced nearly 100fold fewer photons: CBR2/CycLuc1, CBR2/Akalumine$\mathrm{HCl}$, CBG99/Cycluc1, CBG99/ $\mathrm{NH}_{2}-\mathrm{NpLH2}$, and Akaluc/ $\mathrm{NH}_{2}$-NpLH2 (Fig. 4e, Table 1).

\section{Discussion}

A variety of new luciferase enzymes and novel substrate analogues emerging in recent years have resulted in better tools for in vivo BLI. One example is $\mathrm{CBR} 2 / \mathrm{NH}_{2}-\mathrm{NpLH} 2$, which was engineered specifically for enhanced NIR emission to improve imaging resolution in deeper tissues [12]. Another example is Akaluc/Akalumine- $\mathrm{HCl}$ [11], an engineered pair offering improved in vivo sensitivity. Another relatively new substrate, Cycluc1, has shown in vivo utility (including more efficient crossing of the blood brain barrier compared with D-LH2) when used in combination with the already well-established Luc2 [8]. With the emergence of these and other new bioluminescence systems, we felt it would be of interest and potential benefit for the in vivo BLI community, particularly for those interested in dual color readouts, to analyze different pairings of luciferase/substrate using a common set of test parameters. Here, we report on the photon yields and spectral characterization of Luc2, CBG99, CBR2, and Akaluc luciferases combined with four different substrates (D-LH2, $\mathrm{NH}_{2}-\mathrm{NpLH} 2$, Cycluc1, and Akalumine- $\mathrm{HCl}$ ) both in vitro and in vivo. Our goal was to use these parameters to compare the various luciferase/substrate combinations in a standard subcutaneous in vivo BLI model, with the intention to provide guidance for the in vivo BLI community when choosing appropriate systems for specific applications involving dual color detection. Note the longer emission wavelengths for $\mathrm{CBR} 2 / \mathrm{NH}_{2}-$ $\mathrm{NpLH} 2$ and Akaluc/Akalumine-HCl provide a sensitivity advantage in deeper tissue $[11,12]$ that will not be fully realized in a subcutaneous model. However, we postulated that the peak emissions in the NIR for these systems would provide excellent spectral separation from shorter wavelength signals nonetheless.

We have demonstrated in vitro that at a relatively low, but biologically relevant (in vivo) substrate concentration $(0.1 \mathrm{mM})$, three of the four luciferases give maximum signal when combined with D-LH2. The exception was Akaluc, which produced more photons when using either Cyclucl or Akalumine- $\mathrm{HCl}$ as substrate. We observed the same trend in a low-depth, superficial in vivo tissue model. Though we did not examine deeper tissues in this study, we predict based on our results that the red-shifted NIR systems (CBR2/ $/ \mathrm{NH}_{2}-\mathrm{NpLH} 2$, Akaluc/Akalumine- $\mathrm{HCl}$, and Luc2/Akalumine- $\mathrm{HCl}$ ) would perform best.

To evaluate spectral properties in vivo as a way to determine the potential for multiplexing, we used the same superficial, subcutaneous model where different luciferase expressing cell lines were injected into the backs of mice. This minimally invasive 

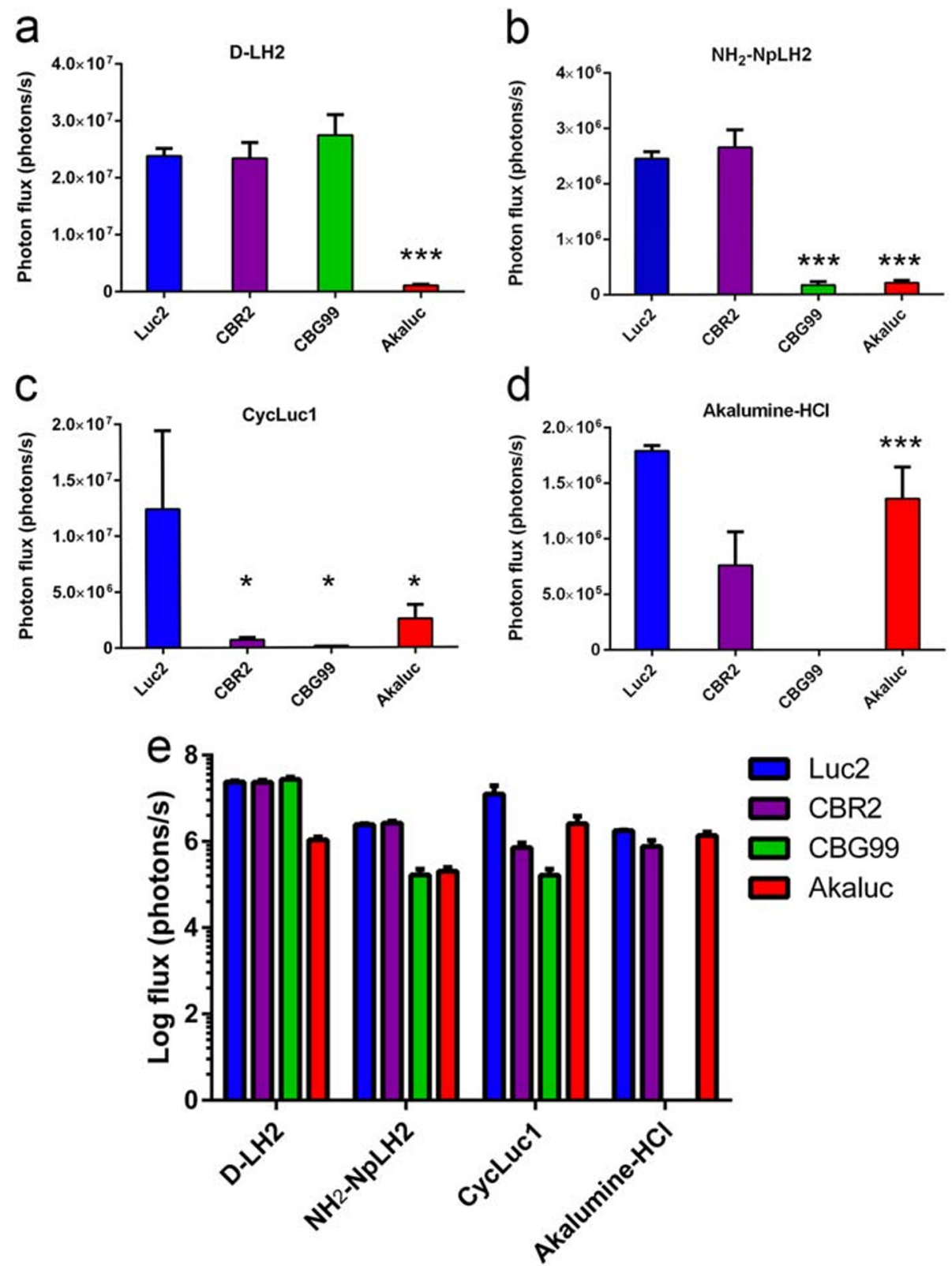

Fig. 4. Quantification of photon flux (ph/s) measured in vivo for all combinations of luciferase and substrate (D-LH2 (a), Akalumine- $\mathrm{HCl}$ (b), CycLuc1 (c), and $\mathrm{NH}_{2}-\mathrm{NpLH} 2$ (d). Combined data is also presented in logarithmic scale (e). Statistical analysis of data was performed using one-way ANOVA followed by Tukey's post-test $\left.{ }^{{ }^{\star \star}} p<0.0019 ;{ }^{* \star} p<0.001 ;{ }^{* \star \star} p<0.0001\right)$.

model allowed us to determine the light emission characteristics for different BLI systems using a small cohort of animals. Based on the analysis, we are able to recommend new combinations of luciferases with distinct colors having potential for multiplexing with a single substrate in superficial tissue e.g., CBG99/D-LH2 $(540 \mathrm{~nm})$ and CBR2/D-LH2 $(620 \mathrm{~nm})$ (examples of spectral unmixing showed in Supplementary Fig. 3); CBG99/D-LH2 $(540 \mathrm{~nm})$ and Luc2/D-LH2 $(610 \mathrm{~nm})$; and Luc2/Akalumine $(680 \mathrm{~nm})$ and Akaluc/Akalumine $(650 \mathrm{~nm})$. Such an approach could be useful for analyzing multiple parameters or biological processes in animals using either engrafted cells or transgenes expressed in particular tissues or organs, and as part of a single imaging session requiring fewer animals.
Successful multiplexing of luminescence systems with different emission spectra relies on the acquisition of images using multiple filters followed by accurate, algorithm-based spectral unmixing to resolve the contributions from each luciferase to total light output. This can be a challenge with shorter wavelength systems (e.g., CBG99/D-LH2), as they tend to shift their apparent emission peak to significantly longer wavelengths when imaged in deeper tissues or even in superficial tissue when using mice with dark fur [20-22]. For these more challenging imaging targets, it is therefore desirable to use bioluminescence pairs that emit in the NIR (> $650 \mathrm{~nm})$, as emission peaks are essentially constant in this range of the spectrum $[22,23]$. In this regard, we found that click beetle luciferases have high photon emission with $\mathrm{NH}_{2}-\mathrm{NpLH} 2$ 
Table 1 In vivo emission intensity relative to Luc2/D-LH2
D-LH2
$\mathrm{NH}_{2}-\mathrm{NpLH} 2$
CycLuc1
Akalumine- $\mathrm{HCl}$

\begin{tabular}{|c|cc|cc|cc|cc|}
\hline Luciferase & $\begin{array}{c}\text { Spectral } \\
\text { peak }[\mathrm{nm}]\end{array}$ & $\begin{array}{c}\text { Normalized } \\
\text { intensity }\end{array}$ & $\begin{array}{c}\text { Spectral } \\
\text { peak [nm] }\end{array}$ & $\begin{array}{c}\text { Normalized } \\
\text { intensity }\end{array}$ & $\begin{array}{c}\text { Spectral } \\
\text { peak [nm] }\end{array}$ & $\begin{array}{c}\text { Normalized } \\
\text { intensity }\end{array}$ & $\begin{array}{c}\text { Spectral } \\
\text { peak [nm] }\end{array}$ & $\begin{array}{c}\text { Normalized } \\
\text { intensity }\end{array}$ \\
\hline Luc2 & 620 & $\mathbf{1}$ & 700 & 0.1 & 620 & 0.5 & 700 & $\mathbf{0 . 8}$ \\
\hline CBR2 & 620 & $\mathbf{0 . 9 8}$ & 720 & 0.1 & 620 & 0.03 & 680 & 0.03 \\
\hline CBG99 & 540 & $\mathbf{1}$ & 620 & 0.01 & 620 & 0.01 & 680 & 0 \\
\hline Akaluc & 640 & 0.05 & 720 & 0.01 & 620 & 0.1 & 660 & 0.06 \\
\hline
\end{tabular}

[12] and that there is a broad spectral separation between CBG99 $(620 \mathrm{~nm})$ and CBR2 (720 nm) (spectral unmixing is shown in Supplementary Fig. 2). However, before giving serious consideration to this pair with $\mathrm{NH}_{2}-\mathrm{NpLH} 2$ as a multiplexing opportunity for deep tissue imaging in mice, it will likely be necessary to improve the photon yield for $\mathrm{CBG} 99 / \mathrm{NH}_{2}-\mathrm{NpLH} 2$.

Funding. This study is funded by the European Commission under the H2020-MSCA-RISE award grant number 777682 (CANCER) and under the H2020-MSCA-ITN award, grant number 675743 (ISPIC), and the Applied Molecular Imaging Erasmus MC (AMIE) facility.

\section{Compliance with Ethical Standards}

\section{Conflict of Interest}

The authors declare that they have no conflict of interest.

Open Access This article is licensed under a Creative Commons Attribution 4.0 International License, which permits use, sharing, adaptation, distribution and reproduction in any medium or format, as long as you give appropriate credit to the original author(s) and the source, provide a link to the Creative Commons licence, and indicate if changes were made. The images or other third party material in this article are included in the article's Creative Commons licence, unless indicated otherwise in a credit line to the material. If material is not included in the article's Creative Commons licence and your intended use is not permitted by statutory regulation or exceeds the permitted use, you will need to obtain permission directly from the copyright holder. To view a copy of this licence, visit http:// creativecommons.org/licenses/by/4.0/.
Publisher's Note. Springer Nature remains neutral with regard to jurisdictional claims in published maps and institutional affiliations.

\section{References}

1. Contag CH, Bachmann M (2002) Advances in in vivo bioluminescence imaging of gene expression. Annu Rev Biomed Eng 4:235-260

2. Brader P, Serganova I, Blasberg RG (2013) Noninvasive molecular imaging using reporter genes. J Nucl Med 54:167-172

3. Close DM, Xu T, Sayler GS, Ripp S (2010) In vivo bioluminescent imaging (BLI): noninvasive visualization and interrogation of biological processes in living animals. Sensors (Basel, Switzerland) 11:180-206

4. Wilson TA, Hastings JW (1998) Bioluminescence. Annu Rev Cell Dev Biol 14:197-230

5. Mezzanotte L, vant Root M, Karatas $\mathrm{H}$ et al (2017) In vivo molecular bioluminescence imaging: new tools and applications. Trends Biotechnol 35:640-652

6. Xu TCD, Handagama W, Marr E et al (2016) The expanding toolbox of in vivo bioluminescent imaging. Front Oncol 6:150

7. Adams ST, Miller SC (2014) Beyond D-luciferin: expanding the scope of bioluminescence imaging in vivo. Curr Opin Chem Biol 21:112-120

8. Evans MS, Chaurette JP, Adams ST et al (2014) A synthetic luciferin improves bioluminescence imaging in live mice. Nat Methods 11:393-395

9. Simonyan H, Hurr C, Young CN (2016) A synthetic luciferin improves in vivo bioluminescence imaging of gene expression in cardiovascular brain regions. Physiol Genomics 48(10):762-770

10. Kuchimaru T, Iwano S, Kiyama M, Mitsumata S, Kadonosono T, Niwa H, Maki S, Kizaka-Kondoh S (2016) A luciferin analogue generating near-infrared bioluminescence achieves highly sensitive deep-tissue imaging. Nat Commun 7:11856

11. Iwano S, Sugiyama M, Hama $\mathrm{H}$, Watakabe A, Hasegawa $\mathrm{N}$, Kuchimaru T, Tanaka KZ, Takahashi M, Ishida Y, Hata J, Shimozono S, Namiki K, Fukano T, Kiyama M, Okano H, Kizaka-Kondoh S, McHugh TJ, Yamamori T, Hioki H, Maki S, Miyawaki A (2018) Single-cell bioluminescence imaging of deep tissue in freely moving animals. Science 359:935-939 
12. Hall MP, Woodroofe CC, Wood MG, Que I, van't Root M, Ridwan Y, Shi C, Kirkland TA, Encell LP, Wood KV, Löwik C, Mezzanotte L (2018) Click beetle luciferase mutant and near infrared naphthylluciferins for improved bioluminescence imaging. Nat Commun 9:132

13. Ohmiya Y (2015) Simultaneous multicolor luciferase reporter assays for monitoring of multiple genes expressions. Comb Chem High Throughput Screen 18:937

14. Branchini BR, Southworth TL, Fontaine DM, Kohrt D, Florentine CM, Grossel MJ (2018) A firefly luciferase dual color bioluminescence reporter assay using two substrates to simultaneously monitor two gene expression events. Sci Rep 8:5990

15. Miloud T, Gunter CH, Hammerling J Quantitative comparison of click beetle and firefly luciferases for in vivo bioluminescence imaging. $\mathrm{J}$ Biomed Opt 12(5)

16. Mezzanotte L, Aswendt M, Tennstaedt A, Hoeben R, Hoehn M, Löwik C (2013) Evaluating reporter genes of different luciferases for optimized in vivo bioluminescence imaging of transplanted neural stem cells in the brain. Contrast Media Mol Imaging 8:505-513

17. Branchini BR, Ablamsky DM, Davis AL, Southworth TL, Butler B, Fan F, Jathoul AP, Pule MA (2010) Red-emitting luciferases for bioluminescence reporter and imaging applications. Anal Biochem 396:290-297
18. Kaskova ZM, Tsarkova AS, Yampolsky IV (2016) 1001 lights: luciferins, luciferases, their mechanisms of action and applications in chemical analysis, biology and medicine. Chem Soc Rev 45:6048-6077

19. Contag CH, Spilman SD, Contag PR, Oshiro M, Eames B, Dennery P, Stevenson DK, Benaron DA (1997) Visualizing gene expression in living mammals using a bioluminescent reporter. Photochem Photobiol 66(4):523-531

20. Mezzanotte L, Que I, Kaijzel E, Branchini B, Roda A, Löwik C (2011) Sensitive dual color in vivo bioluminescence imaging using a new red codon optimized firefly luciferase and a green click beetle luciferase. PLoS One 6. https://doi.org/10.1371/journal.pone.0019277

21. Aswendt M, Vogel S, Schäfer C, Jathoul A, Pule M, Hoehn M (2019) Quantitative in vivo dual-color bioluminescence imaging in the mouse brain. Neurophotonics. 6(2):025006

22. Stowe CL, Burley TA, Allan H, Vinci M, Kramer-Marek G, Ciobota DM, Parkinson GN, Southworth TL, Agliardi G, Hotblack A, Lythgoe MF, Branchini BR, Kalber TL, Anderson JC, Pule MA (2019) Nearinfrared dual bioluminescence imaging in mouse models of cancer using infraluciferin. Elife 8. https://doi.org/10.7554/eLife.45801

23. Jathoul A, Grounds E, Anderson JC, Pule M (2015) A dual-color far-red to near-infrared firefly luciferin analogue designed for multiparametric bioluminescence imaging. Angew Chem Int Ed Eng 54(6):1698

Publisher's Note Springer Nature remains neutral with regard to jurisdictional claims in published maps and institutional affiliations. 